\title{
Convolution conditions for bounded $\alpha$-starlike functions of complex order
}

\begin{abstract}
Let $A$ be the class of analytic functions in the unit disc $U$ of the complex plane $\mathbb{C}$ with the normalization $f(0)=f^{\prime}(0)-1=0$. We introduce a subclass $S_{M}^{*}(\alpha, b)$ of $A$, which unifies the classes of bounded starlike and convex functions of complex order. Making use of Salagean operator, a more general class $S_{M}^{*}(n, \alpha, b)(n \geq 0)$ related to $S_{M}^{*}(\alpha, b)$ is also considered under the same conditions. Among other things, we find convolution conditions for a function $f \in A$ to belong to the class $S_{M}^{*}(\alpha, b)$. Several properties of the class $S_{M}^{*}(n, \alpha, b)$ are investigated.
\end{abstract}

1. Introduction. Let $H$ denote the class of analytic functions in the unit disc $U=\{z \in \mathbb{C}:|z|<1\}$. Let $A$ denote the subclass of $H$ consisting of functions of the form

$$
f(z)=z+\sum_{k=2}^{\infty} a_{k} z^{k} \quad(z \in U) .
$$

For functions $f$ given by (1.1) and $g \in A$ defined by $g(z)=z+\sum_{k=2}^{\infty} b_{k} z^{k}$, $z \in U$, the Hadamard product (or convolution) of $f$ and $g$ is given by

$$
(f * g)(z)=z+\sum_{k=2}^{\infty} a_{k} b_{k} z^{k} \quad(z \in U) .
$$

2010 Mathematics Subject Classification. 30C45, 30C50.

Key words and phrases. Univalent functions, bounded starlike functions of complex order, bounded convex functions of complex order, $\alpha$-starlike functions. 
Let $\Omega$ be a family of functions $\omega$ which are analytic in $U$ and satisfy the conditions $\omega(0)=0,|\omega(z)|<1$, for every $z \in U$. Given real number $M$, $M>\frac{1}{2}$, let $S_{M}^{*}$ be the class of bounded starlike functions $f \in A$ satisfying the condition

$$
\left|\frac{z f^{\prime}(z)}{f(z)}-M\right| \leq M \quad(z \in U) .
$$

This class was introduced and studied by Singh and Singh [16].

We say that $f \in A$ belongs to the class $F(b, M)\left(b \in \mathbb{C}^{*}=\mathbb{C} \backslash\{0\}, M>\frac{1}{2}\right)$ of bounded starlike functions of complex order, if and only if $\frac{f(z)}{z} \neq 0$ in $U$ and

$$
\left|\frac{b-1+\frac{z f^{\prime}(z)}{f(z)}}{b}-M\right|<M \quad(z \in U) .
$$

The class $F(b, M)$ was introduced by Nasr and Aouf [9]. Let $C(b, M)(b \in$ $\mathbb{C}^{*}, M>\frac{1}{2}$ ) be the class of bounded convex functions of complex order, i.e., of functions $f \in A$ such that

$$
z f^{\prime}(z) \in F(b, M) .
$$

This class $C(b, M)$ was introduced and studied by Nasr and Aouf [8].

First let us define the class $S_{M}^{*}(\alpha, b)$ which unifies the classes of bounded starlike and convex functions of complex order.

Definition 1. We say that $f \in A$ belongs to the class $S_{M}^{*}(\alpha, b)\left(b \in \mathbb{C}^{*}\right.$, $\alpha \geq 0, M>\frac{1}{2}$ ) of bounded $\alpha$-starlike functions of complex order, if and only if $\frac{f(z) f^{\prime}(z)}{z} \neq 0$ in $U$ and

$$
\left|1+\frac{1}{b}\left(\frac{(1-\alpha) z f^{\prime}(z)+\alpha z\left(z f^{\prime}(z)\right)^{\prime}}{(1-\alpha) f(z)+\alpha z f^{\prime}(z)}-1\right)-M\right|<M \quad(z \in U) .
$$

One can easily show that $f \in S_{M}^{*}(\alpha, b)$ if and only if there is a function $g \in S_{M}^{*}$ such that

$$
(1-\alpha) f(z)+\alpha z f^{\prime}(z)=z\left(\frac{g(z)}{z}\right)^{b} \quad(z \in U) .
$$

It was shown in [16] that $g \in S_{M}^{*}$ if and only if for $z \in U$

$$
\frac{z g^{\prime}(z)}{g(z)}=\frac{1+\omega(z)}{1-m \omega(z)}, \quad m=1-\frac{1}{M}
$$

for some $\omega \in \Omega$. Thus from (1.3) and (1.4) follows that $f \in S_{M}^{*}(\alpha, b)$ if and only if

$$
\frac{(1-\alpha) z f^{\prime}(z)+\alpha z\left(z f^{\prime}(z)\right)^{\prime}}{(1-\alpha) f(z)+\alpha z f^{\prime}(z)}=\frac{1+[b(1+m)-m] \omega(z)}{1-m \omega(z)} \quad(z \in U) .
$$


Taking specific values of $\alpha, b$ and $M$, we obtain the following subclasses studied by various authors:

(1) $S_{M}^{*}(0, b) \equiv F(b, M)$ and $S_{M}^{*}(1, b) \equiv C(b, M)$.

(2) $S_{M}^{*}\left(0, e^{-i \lambda} \cos \lambda\right) \equiv F_{\lambda, M}\left(|\lambda|<\frac{\pi}{2}\right)$ is the class of bounded $\lambda$ spirallike functions and $S_{M}^{*}\left(1, e^{-i \lambda} \cos \lambda\right) \equiv C_{\lambda, M}\left(|\lambda|<\frac{\pi}{2}\right)$ is the class of bounded Robertson functions that satisfy the condition $z f^{\prime}(z) \in F_{\lambda, M}$, which were studied by Kulshrestha [4].

(3) $S_{M}^{*}(0,1) \equiv S_{M}^{*}$ is the class of bounded starlike functions.

(4) $S_{\infty}^{*}\left(0,(1-\alpha) e^{-i \lambda} \cos \lambda\right) \equiv S_{\lambda}(\alpha)\left(|\lambda|<\frac{\pi}{2}, 0 \leq \alpha<1\right)$ is the class of $\lambda$-spirallike functions of order $\alpha$ (see Libera [6]) and $S_{\infty}^{*}(1,(1-$ $\left.\alpha) e^{-i \lambda} \cos \lambda\right) \equiv C_{\lambda}(\alpha)\left(|\lambda|<\frac{\pi}{2}, 0 \leq \alpha<1\right)$ (see Kulshrestha [5] and Sizuk [15]).

(5) $S_{\infty}^{*}(0, b) \equiv S(b)$, is the class of starlike functions of complex order (see Nasr and Aouf [10]).

(6) $S_{\infty}^{*}(1, b) \equiv C(b)$ is the class of convex functions of complex order (see Wiatrowski [17] and Nasr and Aouf [7]).

(7) $S_{\infty}^{*}(0,1-\alpha) \equiv S^{*}(\alpha)(0 \leq \alpha<1)$ is the class of starlike functions of order $\alpha$ and $S_{\infty}^{*}(1,1-\alpha)=C(\alpha)(0 \leq \alpha<1)$ is the class of convex functions of order $\alpha$ (see Robertson [12]).

(8) $S_{\infty}^{*}(0,1) \equiv S^{*}, S_{\infty}^{*}(1,1) \equiv C$ and $S_{\infty}^{*}\left(0, e^{-i \lambda} \cos \lambda\right) \equiv S_{\lambda}\left(|\lambda|<\frac{\pi}{2}\right)$ are the classes of starlike, convex and spirallike functions (More about these classes one can see in the Goodman's book [3]).

For $f \in A$, Salagean [13] introduced the following operator $D^{n} f(n \in$ $\left.\mathbb{N}_{0}=\mathbb{N} \cup\{0\}=\{0,1,2,3, \ldots\}\right)$ which is called the Salagean operator:

$$
\begin{aligned}
& D^{0} f(z)=f(z), D^{1} f(z)=D f(z)=z f^{\prime}(z), \\
& D^{n} f(z)=D\left(D^{n-1} f(z)\right) \quad(z \in U) .
\end{aligned}
$$

From the definition of $D^{n} f$ it follows at once that

$$
D^{n} f(z)=z+\sum_{k=2}^{\infty} k^{n} a_{k} z^{k} \quad(z \in U) .
$$

With the aid of Salagean operator, we introduce the class $S_{M}^{*}(n, \alpha, b)$ as follows:

Definition 2. Let $M>\frac{1}{2}, b \in C^{*}, \alpha \geq 0$ and $n \in \mathbb{N}_{0}$. A function $f \in A$ is said to be in the class $S_{M}^{*}(n, \alpha, b)$ if and only if,

$$
\left|1+\frac{1}{b}\left(\frac{(1-\alpha) D^{n+1} f(z)+\alpha D^{n+2} f(z)}{(1-\alpha) D^{n} f(z)+\alpha D^{n+1} f(z)}-1\right)-M\right|<M \quad(z \in U) .
$$

We note that $S_{M}^{*}(n, 0, b) \equiv H_{n}(b, M)$ which was studied by Aouf et al. [1].

The object of the present paper is to investigate some convolution properties of the class $S_{M}^{*}(\alpha, b)$. Using these properties, we obtain the necessary and sufficient condition for $f \in A$ to belong to the class $S_{M}^{*}(n, \alpha, b)$. Also we 
establish the relationship among the classes $S_{M}^{*}(n+1, \alpha, b)$ and $S_{M}^{*}(n, \alpha, b)$. These results generalize the related works of some authors.

2. Convolution conditions. Unless otherwise mentioned, we assume throughout this article that $b \in \mathbb{C}^{*}, M>\frac{1}{2}, \alpha \geq 0$ and $n \in \mathbb{N}_{0}$.

Theorem 1. A function $f$ of the form (1.1) is in the class $S_{M}^{*}(\alpha, b)$ if and only if

$$
\frac{1}{z}\left[f(z) *\left\{(1-\alpha) \frac{z-C z^{2}}{(1-z)^{2}}+\alpha \frac{z+(1-2 C) z^{2}}{(1-z)^{3}}\right\}\right] \neq 0 \quad(z \in U)
$$

where $C=C_{\theta}=\frac{e^{-i \theta}+[b(1+m)-m]}{b(1+m)}, \theta \in[0,2 \pi)$.

Proof. A function $f$ is in the class $S_{M}^{*}(\alpha, b)$ if and only if

$$
\frac{(1-\alpha) z f^{\prime}(z)+\alpha z\left(z f^{\prime}(z)\right)^{\prime}}{(1-\alpha) f(z)+\alpha z f^{\prime}(z)}=\frac{1+[b(1+m)-m] \omega(z)}{1-m \omega(z)} \quad(z \in U),
$$

where $m=1-\frac{1}{M}$, which is equivalent to

$$
\frac{z\left[(1-\alpha) f(z)+\alpha z f^{\prime}(z)\right]^{\prime}}{(1-\alpha) f(z)+\alpha z f^{\prime}(z)} \neq \frac{1+[b(1+m)-m] e^{i \theta}}{1-m e^{i \theta}}
$$

$(z \in U, \theta \in[0,2 \pi))$ and further to

$$
\begin{aligned}
& z\left[(1-\alpha) f(z)+\alpha z f^{\prime}(z)\right]^{\prime}\left(1-m e^{i \theta}\right) \\
& \quad-\left[(1-\alpha) f(z)+\alpha z f^{\prime}(z)\right]\left(1+[b(1+m)-m] e^{i \theta}\right) \neq 0
\end{aligned}
$$

for some $z \in U$ and $\theta \in[0,2 \pi)$. It is well known that

$$
f(z)=f(z) * \frac{z}{(1-z)}, z f^{\prime}(z)=f(z) * \frac{z}{(1-z)^{2}} \quad(z \in U) .
$$

Using (2.4), it is easy to verify that

$$
(1-\alpha) f(z)+\alpha z f^{\prime}(z)=f(z) * \frac{z-(1-\alpha) z^{2}}{(1-z)^{2}} \quad(z \in U) .
$$

Since $z(f * g)^{\prime}=f * z g^{\prime}$, we have

$$
z\left[(1-\alpha) f(z)+\alpha z f^{\prime}(z)\right]^{\prime}=f(z) * \frac{z+(2 \alpha-1) z^{2}}{(1-z)^{3}} \quad(z \in U) .
$$

Substituting (2.5) and (2.6) into (2.3), we get

$$
\begin{aligned}
\frac{1}{z}[f(z) & *\left\{-(1-\alpha)(1-z)\left[b(1+m) e^{i \theta} z\right.\right. \\
& \left.-\left(1+[b(1+m)-m] e^{i \theta}\right) z^{2}\right] \\
& \left.\left.-\alpha(1-z) b(1+m) e^{i \theta} z+2 \alpha\left(1-m e^{i \theta}\right) z^{2}\right\} /(1-z)^{3}\right] \neq 0
\end{aligned}
$$


$(z \in U, \theta \in[0,2 \pi))$ i.e., equivalently,

$$
\begin{aligned}
\frac{1}{z}[f(z) & *\left\{-(1-\alpha)(1-z)\left[b(1+m) e^{i \theta} z-\left(1+[b(1+m)-m] e^{i \theta}\right) z^{2}\right]\right. \\
& -\alpha\left[b(1+m) e^{i \theta} z+\left\{b(1+m) e^{i \theta}\right.\right. \\
& \left.\left.\left.\left.-2\left(1+[b(1+m)-m] e^{i \theta}\right)\right\} z^{2}\right]\right\} /(1-z)^{3}\right] \neq 0
\end{aligned}
$$

for some $z \in U$ and $\theta \in[0,2 \pi)$. Thus (2.7) can be rewritten as follows

$$
\begin{aligned}
\frac{1}{z}[f(z) & *\left\{(1-\alpha) \frac{z-\frac{e^{-i \theta}+[b(1+m)-m]}{b(1+m)} z^{2}}{(1-z)^{2}}\right. \\
+ & \left.\left.\alpha \frac{z+\left(1-2 \frac{e^{-i \theta}+[b(1+m)-m]}{b(1+m)}\right) z^{2}}{(1-z)^{3}}\right\}\right] \neq 0
\end{aligned}
$$

where $z \in U, \theta \in[0,2 \pi)$. Hence the proof of Theorem 1 is complete.

\section{Remark 1.}

(1) Taking $\alpha=0$ in Theorem 1, we obtain the result obtained by ElAshwah [2, Theorem 2.1].

(2) Taking $\alpha=1$ in Theorem 1, we obtain the result obtained by ElAshwah [2, Theorem 2.4].

(3) Taking $\alpha=1, b=1-\beta(0 \leq \beta<1), M=\infty$ and $e^{i \theta}=x$ in Theorem 1, we obtain the result obtained by Silverman et al. [14, Theorem 1].

(4) Taking $\alpha=0, b=1-\beta(0 \leq \beta<1), M=\infty$ and $e^{i \theta}=x$ in Theorem 1 , we obtain the result obtained by Silverman et al. [14, Theorem 2].

(5) Taking $\alpha=1, b=e^{-i \lambda} \cos \lambda(|\lambda|<1), M=\infty$ and $e^{i \theta}=x$ in Theorem 1, we obtain the result obtained by Padmanabhan and Ganesan [11, Theorem 1] with $B=-1$ and $A=1$.

(6) Taking $\alpha=0, b=e^{-i \lambda} \cos \lambda(|\lambda|<1), M=\infty$ and $e^{i \theta}=x$ in Theorem 1, we obtain the result obtained by Padmanabhan and Ganesan [11, Theorem 2] with $B=-1$ and $A=1$.

Theorem 2. A function $f$ of the form (1.1) is in the class $S_{M}^{*}(n, \alpha, b)$ if and only if

$$
1-\sum_{k=2}^{\infty} k^{n} \frac{(k-1)\left[e^{-i \theta}-m\right]-b(1+m)}{b(1+m)}[(1-\alpha)+\alpha k] a_{k} z^{k-1} \neq 0
$$

for all $\theta \in[0,2 \pi)$ and $z \in U$. 
Proof. Note that $f \in S_{M}^{*}(n, \alpha, b)$ if and only if $D^{n} f \in S_{M}^{*}(\alpha, b)$. Thus from Theorem 1 , we have $f \in S_{M}^{*}(n, \alpha, b)$ if and only if

(2.9) $\frac{1}{z}\left[D^{n} f(z) *\left\{(1-\alpha) \frac{z-C z^{2}}{(1-z)^{2}}+\alpha \frac{z+(1-2 C) z^{2}}{(1-z)^{3}}\right\}\right] \neq 0 \quad(z \in U)$

where $C=C_{\theta}=\frac{e^{-i \theta}+[b(1+m)-m]}{b(1+m)}$ and $\theta \in[0,2 \pi)$, i.e., if and only if

$$
\begin{aligned}
\frac{1}{z}\left[D^{n} f(z)\right. & *\left\{(1-\alpha)\left[\frac{C z}{1-z}+\frac{(1-C) z}{(1-z)^{2}}\right]\right. \\
+ & \left.\left.\alpha\left[\frac{2(1-C) z}{(1-z)^{3}}-\frac{(1-2 C) z}{(1-z)^{2}}\right]\right\}\right] \neq 0
\end{aligned}
$$

$(z \in U)$. Since for $z \in U$,

$$
\frac{z}{1-z}=z+\sum_{k=2}^{\infty} z^{k}, \quad \frac{z}{(1-z)^{2}}=z+\sum_{k=2}^{\infty} k z^{k}
$$

and

$$
\frac{z}{(1-z)^{3}}=z+\sum_{k=2}^{\infty} \frac{k(k+1)}{2} z^{k}
$$

from (1.6) and (2.10) it follows that

$$
1-\sum_{k=2}^{\infty} k^{n} \frac{(k-1)\left[e^{-i \theta}-m\right]-b(1+m)}{b(1+m)}[(1-\alpha)+\alpha k] a_{k} z^{k-1} \neq 0
$$

$(z \in U)$. This completes the proof of Theorem 2 .

Theorem 3. If $f \in A$ satisfies the inequality

$$
\sum_{k=2}^{\infty}(k-1+|b|)[(1-\alpha)+\alpha k] k^{n}\left|a_{k}\right| \leq|b|,
$$

then $f \in S_{M}^{*}(n, \alpha, b)$.

Proof. Since

so

$$
\left|\frac{(k-1)\left[e^{-i \theta}-m\right]-b(1+m)}{b(1+m)}\right| \leq \frac{(k-1+|b|)}{|b|},
$$

$$
\begin{aligned}
& \left|1-\sum_{k=2}^{\infty} \frac{(k-1)\left[e^{-i \theta}-m\right]-b(1+m)}{b(1+m)}[(1-\alpha)+\alpha k] k^{n} a_{k} z^{k-1}\right| \\
& \geq 1-\sum_{k=2}^{\infty}\left|\frac{(k-1)\left[e^{-i \theta}-m\right]-b(1+m)}{b(1+m)}\right|[(1-\alpha)+\alpha k] k^{n}\left|a_{k}\right||z|^{k-1} \\
& \geq 1-\sum_{k=2}^{\infty} \frac{k-1+|b|}{|b|}[(1-\alpha)+\alpha k] k^{n}\left|a_{k}\right|>0
\end{aligned}
$$


$(z \in U)$. Thus (2.8) holds, which ends the proof.

Theorem 4. $S_{M}^{*}(n+1, \alpha, b) \subset S_{M}^{*}(n, \alpha, b)$.

Proof. Let $f \in S_{M}^{*}(n+1, \alpha, b)$. By Theorem 2, we have

$$
1-\sum_{k=2}^{\infty} \frac{(k-1)\left[e^{-i \theta}-m\right]-b(1+m)}{b(1+m)}[(1-\alpha)+\alpha k] k^{n+1} a_{k} z^{k-1} \neq 0
$$

$(z \in U)$, which is equivalent to

$$
\begin{aligned}
& {\left[1+\sum_{k=2}^{\infty} k z^{k-1}\right]} \\
& *\left[1-\sum_{k=2}^{\infty} k^{n} \frac{(k-1)\left[e^{-i \theta}-m\right]-b(1+m)}{b(1+m)}[(1-\alpha)+\alpha k] a_{k} z^{k-1}\right] \neq 0
\end{aligned}
$$

$(z \in U)$. Since

$$
\left[1+\sum_{k=2}^{\infty} k z^{k-1}\right] *\left[1+\sum_{k=2}^{\infty} \frac{1}{k} z^{k-1}\right]=1+\sum_{k=2}^{\infty} z^{k-1} \quad(z \in U),
$$

by using the property, if $f \neq 0$ and $g * h \neq 0$, then $f *(g * h) \neq 0,(2.13)$ can be written as

$$
1-\sum_{k=2}^{\infty} k^{n} \frac{(k-1)\left[e^{-i \theta}-m\right]-b(1+m)}{b(1+m)}[(1-\alpha)+\alpha k] a_{k} z^{k-1} \neq 0
$$

$(z \in U)$. Thus the assertion follows from Theorem 2 .

(1) Putting $\alpha=0$ in Theorems 2, 3 and 4, we get the results obtained by El-Ashwah [2, Theorems 2.7, 3.1 and 3.4].

(2) Putting $\alpha=1$ in Theorems 2, 3 and 4, we get the results obtained by El-Ashwah [2, Theorems 2.8, 3.2 and 3.5].

Acknowledgement. The author warmly thanks the referee for his careful reading and making some valuable comments which have essentially improved the presentation of this paper.

\section{REFERENCES}

[1] Aouf, M. K., Darwish, H. E., Attiya, A. A., On a class of certain analytic functions of complex order, Indian J. Pure Appl. Math. 32 (10) (2001), 1443-1452.

[2] El-Ashwah, R. M., Some convolution and inclusion properties for subclasses of bounded univalent functions of complex order, Thai J. Math. 12 (2) (2014), 373384.

[3] Goodman, A. W., Univalent Functions, Vol. I, II, Tampa, Florida, 1983.

[4] Kulshrestha, P. K., Bounded Robertson functions, Rend. Mat. 6 (1) (1976), 137-150.

[5] Kulshrestha, P. K., Distortion of spiral-like mapping, Proc. Royal Irish Acad. 73A (1973) 1-5.

[6] Libera, R. J., Univalent $\lambda$-spiral functions, Canad. J. Mat. 19 (1967) 449-456. 
[7] Nasr, M. A., Aouf, M. K., On convex functions of complex order, Mansoura Sci. Bull. 9 (1982), 565-582.

[8] Nasr, M. A., Aouf, M. K., Bounded convex functions of complex order, Mansoura Sci. Bull. 10 (1983), 513-526.

[9] Nasr, M. A., Aouf, M. K., Bounded starlike functions of complex order, Proc. Indian Acad. Sci. (Math. Sci.) 92 (2) (1983) 97-102.

[10] Nasr, M. A., Aouf, M. K., Starlike functions of complex order, J. Natur. Sci. Math. 25 (1985), 1-12.

[11] Padmanabhan, K. S., Ganesan, M. S., Convolution conditions for certain class of analytic functions, Indian J. Pure Appl. Math. 15 (1984), 777-780.

[12] Robertson, M. S., On the theory of univalent functions, Ann. of Math. 37 (1936), 374-408.

[13] Salagean, G. S., Subclasses of univalent functions, in: Complex Analysis - Fifth Romanian-Finnish Seminar (C. A. Cazacu, N. Boboc, M. Jurchescu, I. Suciu, eds.) Springer, Berlin-Heidelberg, 1983, 362-372.

[14] Silverman, H., Silvia, E. M., Telage, D., Convolution conditions for convexity and starlikeness and spiral-likeness, Math. Z. 162 (1978), 125-130.

[15] Sizuk, P. I., Regular functions $f(z)$ for which $z f^{\prime}(z)$ is $\lambda$-spirallike, Proc. Amer. Math. Soc. 49 (1975), 151-160.

[16] Singh, R., Singh, V., On a class of bounded starlike functions, Indian J. Pure Appl. Math. 5 (8) (1974), 733-754.

[17] Wiatrowski, P., The coefficient of a certain family of holomorphic functions, Zeszyty Nauk. Uniw. Łódz. Nauki Mat. Przyrod. Ser. II No. 39 Mat. (1971), 75-85.

\author{
A. Y. Lashin \\ Department of Mathematics \\ Faculty of Science \\ Mansoura University \\ Mansoura, 35516 \\ Egypt \\ e-mail: aylashin@mans.edu.eg
}

Received June 1, 2016 